\title{
NEOS Detector for Reactor Antineutrinos
}

\author{
Kyung-Kwang Joo, Ba-Ro Kim
}

Department of Physics, Chonnam National University, Gwangju 61186, Korea

E-mail: kvungkwangjoodgmail.com gkfh85dgmail.com

Chang-Hwan Jang, Youngju Ko*, Kim Siyeon

Department of Physics, Chung-Ang University, Seoul 06974, Korea

E-mail: iji8145dgmail.com, godpapa7dgmail.com siveondcau.ac.kn

\section{Eun-Ju Jeon, Young-Duk Kim, Jaison Lee, Moo-Hyun Lee, Yoo-Min Oh, Hyang-Kyu} Park, Kang-Soon Park

Center for Underground Physics, Institute for Basic Science, Daejeon 34047, Korea

E-mail:ejjeondibs.re.kn, vakimdibs.re.kn bisahnleedgmail.com,

moohyun. Leedgmail.com ohyoumeandgmail.com hkparkdibs.re.kr,

hepparkegmail.com

\section{Bo-Young Han, Gwang-Min Sun}

Neutron Science Division, Korea Atomic Energy Research Institute, Daejeon 34057, Korea

E-mail: byhandkaeri.re.kr, gmsundkaeri.re.kr

\section{Hong-Joo Kim, Joo-Young Lee}

Department of Physics, Kyungpook National University, Daegu 41566, Korea

hong jooknudgmail.com bylee8875dgmail.com

\section{Hyunsoo Kim, Jinyu Kim, Kyung-Min Seo}

Department of Physics and Astronomy, Sejong University, Seoul 05006, Korea

E-mail: trilobite333dgmail.com kazides59damail.com

sukm2000dgmail.com

NEOS intends to understand the short-distance behavior of reactor neutrino oscillation. The detector has been obtaining about 2,000 inverse beta decay (IBD) events on daily base, since its performance was stabilized in September 2015. We present the geometry, the structure, and the current output of NEOS detector. The design of detector and the recipe of liquid scintillator mixture will be introduced, which were well set up enough to reduce the level of background. The signal-to-background ratio reached over 20. Including adjustment procedures for reconstruction, we will show the energy resolution of the detector and the energy calibration with GEANT4 simulation.

38th International Conference on High Energy Physics

3-10 August 2016

Chicago, USA

\footnotetext{
* Speaker.
} 


\section{Experimental Site and Detector Geometry}

The NEOS detector is installed at tendon gallery of Reactor Unit 5 in Hanbit Nuclear Power Plant located Yeonggwang, South Korea. The reactor core has the thermal power of $2.8 \mathrm{GW}_{\text {th }}$, the diameter of $3.1 \mathrm{~m}$, and the height of $3.8 \mathrm{~m}$. The distance between reactor core and the detector is about $24 \mathrm{~m}$ and the overburden is about 20 meter water equivalent.

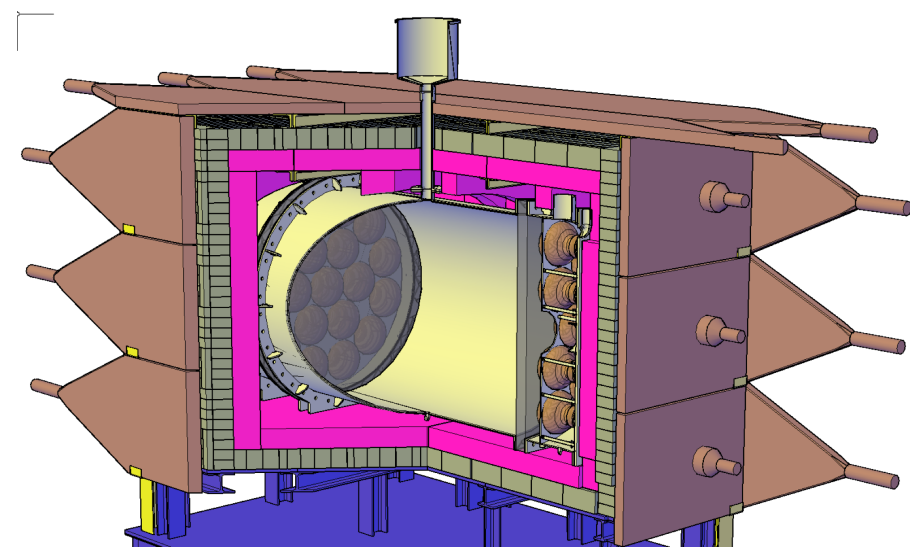

Figure 1: The NEOS detector.

The detector consists of a active target, two buffer tanks, shieldings, and muon detectors as shown in Figure $\mathbb{W}$. The active target is contained in cylindrical tank which has the volume of 1,000 $\mathrm{L}$ and $0.5 \%$ gadolinium loaded liquid scintillator (LS). The LS based the linear alkyl benzene (LAB) and the di-isopropylnaphthalene (DIN) are mixed in the ratio 9:1 by mass.[W] There are two buffer tanks which are filled with mineral oil at both sides of the target and nineteen 8-inch photomultiplier tubes (R5912; Hamamatsu) are installed in each buffer tank. These target and buffer tank are surrounded by shieldings of $10 \mathrm{~cm}$ thick borated polyethylene and $10 \mathrm{~cm}$ thick lead against neutrons and external gammas, respectively. Muon detectors are made with fifteen plastic scintillator plates, twelve 5-inch (R877-100; Hamamatsu), and eighteen 3-inch (H7195; Hamamatsu) photomultiplier tubes.

\section{Monte-Carlo Simulation and Detector Responses}

The detector and its response are studied through the comparison real data and simulated data. As a result, the simulated data is with real data in good agreement as shown in Figure $\square$. In top figures, the response about detector geometry and escaping gamma is well described with simulation and the simulation can be explain in whole energy range for the analysis of reactor neutrino.

Pulse shape discrimination [[] (PSD) via the waveform analysis is used for reducing backgrounds and backgrounds are reduced more than $70 \%$. The ratio of signal to background is about 23 after PSD and the energy resolution is $\sigma / E=\sqrt{0.042^{2} / E+0.021^{2}+0.010 / E^{2}}$ [B]]. Energy is calibrated with the non-linearity between the charge to energy ratio and energy. The relation of the 

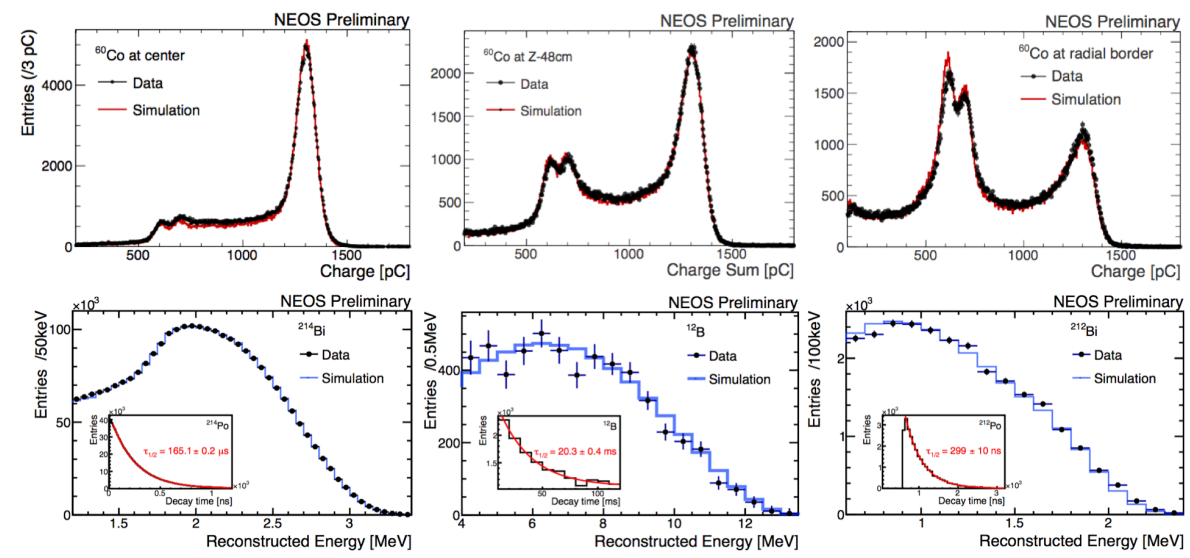

Figure 2: Charge or energy spectrum of beta and gamma sources. Three top figures are ${ }^{60} \mathrm{Co}$ with various positions of source. Bottom figures are beta spectrums and small plot in each figure shows decay time. Left, middle, and right show ${ }^{214} B i,{ }^{12} B$, and ${ }^{212} B i$ in bottom figures, respectively. Black dots and red lines (for top) or blue lines (for bottom) are real data and simulated data.

charge and the temperature is checked and the charge is corrected with this relation for improvement of the energy resolution. The number of IBD is about 2,000 (81) in reactor-on (off) period and the ramping up step can be confirmed with data as shown in Figure [ 3 .

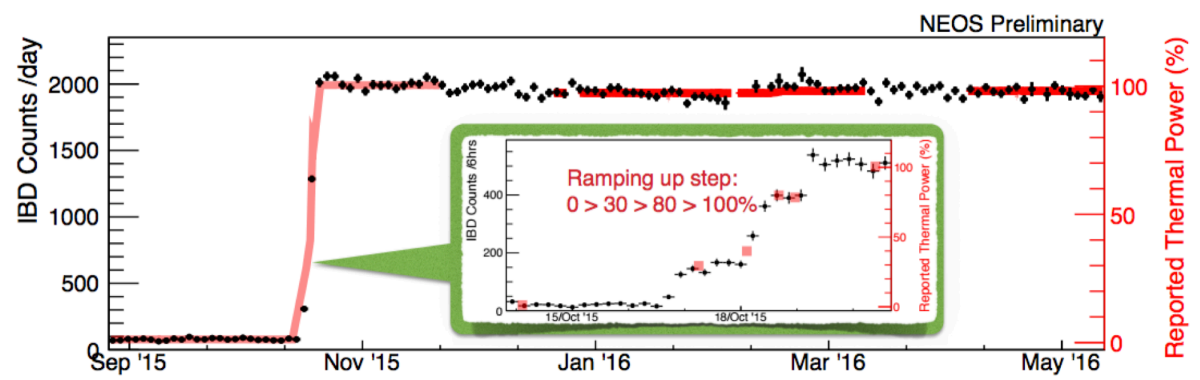

Figure 3: The number of IBD candidates included backgrounds in time.

\section{References}

[1] B. R. Kim et al. [NEOS Collaboration], Pulse Shape Discrimination Capability of Metal-loaded Organic Liquid Scintillators for a Short Baseline Reactor Neutrino Experiment Phys. Scr 90, 055302 (2015).

[2] G. Ranucci, Pulse Shape Discrimination of Liquid Scintilators Nucl. Instrum. Meth. A 354, 389 (1995).

[3] J. Beringer et al., Review of Particle Physics, Phys. Rev. D 86, 010001 (2012). 